\title{
Statistical Survey of Type III Radio Bursts at Long Wavelengths Observed by the Solar TErrestrial RElations Observatory (STEREO)/Waves Instruments: Radio Flux Density Variations with Frequency
}

\author{
V. $\operatorname{Krupar}^{1} \cdot$ M. Maksimovic ${ }^{2}$. \\ O. Santolik ${ }^{1,3}$. E. P. Kontar ${ }^{4}$. B. Cecconi ${ }^{2}$. \\ S. Hoang ${ }^{2}$. O. Kruparova ${ }^{1}$. J. Soucek ${ }^{1}$. \\ H. Reid ${ }^{4}$ A. Zaslavsky ${ }^{2}$ \\ (C) Springer $\bullet \bullet \bullet$
}

\begin{abstract}
We have performed a statistical study of 152 Type III radio bursts observed by Solar TErrestrial RElations Observatory (STEREO)/Waves between May 2007 and February 2013. We have investigated the flux density between $125 \mathrm{kHz}$ and $16 \mathrm{MHz}$. Both high- and low-frequency cutoffs have been observed in $60 \%$ of events suggesting an important role of propagation. As already reported by previous authors, we observed that the maximum flux density occurs at $1 \mathrm{MHz}$ on both spacecraft. We have developed a simplified analytical model of the flux density as a function of radial distance and compared it to the STEREO/ Waves data.
\end{abstract}

Keywords: Solar radio emissions, Plasma radiation

\section{Introduction}

Type III radio bursts are consequence of suprathermal electrons accelerated during solar flares (Wild, 1950). The Type III-generating electron beam propagates outward from the Sun along an open magnetic field line in the interplanetary (IP) medium with speed ranging from $\approx 0.3 \mathrm{c}$ to $\approx 0.05 \mathrm{c}$ (Dulk et al., 1987; Mel'nik, Lapshin, and Kontar, 1999, Dulk, 2000). As the electron beam produces the bump-on-tail instability, it locally excites intense Langmuir waves at

\footnotetext{
1 Institute of Atmospheric Physics ASCR, Bocni II 1403, Prague 141 31, Czech Republic email: vk@ufa.cas.cz

2 LESIA, UMR CNRS 8109, Observatoire de Paris, Meudon

92195, France

3 Faculty of Mathematics and Physics, Charles University,

Ke Karlovu 3, Prague 121 16, Czech Republic

4 Department of Physics and Astronomy, University of

Glasgow, Glasgow G12 8QQ, UK
} 
the local electron plasma frequency $\left[f_{\mathrm{pe}}\right]$. These waves then convert to electromagnetic emission via a series of non-linear processes which are still debated and require better understanding (Melrose, 1980: Robinson and Cairns, 1998).

The generation of Langmuir waves by the beam also produces back-reaction on the local distribution of electrons, which makes the problem of electron propagation inherently non-linear. The characteristic scales of electron-beam propagation $(\approx 1 \mathrm{AU})$ require additional simplifications such as quasi-linear treatment (Drummond and Pines, 1962, Vedenov, Velikhov, and Sagdeev, 1962 Pines and Schrieffer, 1962). The relatively low level of the waves is normally justified by the observations of the mean electric fields associated with Type III exciters. The numerical simulations of transport and generation as well as reabsorption of Langmuir waves show that the electron beam can propagate over large distances with relatively weak losses while at the same time producing a high level of plasma waves locally (Magelssen and Smith, 1977). The important aspect of beam-Langmuir wave evolution is the sensitivity to plasma-density fluctuations in the solar corona and the heliosphere. The density fluctuations can effectively influence the distribution of waves, which in turn affects the electron distribution (Smith and Sime, 1979, Melrose, Cairns, and Dulk, 1986). Numerical simulations (Kontar, 2001) also reveal that the plasma inhomogeneities change the spatial distribution of the waves, while the local electron distribution is weakly affected. However, the effects of density fluctuations and large-scale density gradient in the solar plasma becomes noticeable at large distances (Kontar and Reid, 2009), so that initially injected power-law spectrum becomes a broken one. Overall, the rate at which plasma waves are induced by an unstable electron beam is reduced by background density fluctuations, most acutely when fluctuations have large amplitudes or small wavelengths. Numerical simulations (Reid and Kontar, 2010) further show a direct correlation between the spectrum of the double power-law below the break energy and the turbulent intensity of the background plasma.

Langmuir waves can be converted via the plasma emission process (Ginzburg and Zhelezniakov, 1958) into electromagnetic radiation: Type III radio bursts either at $f_{\mathrm{pe}}$ [the fundamental $[F]$ component], and/or at $2 f_{\mathrm{pe}}$ [the harmonic $[H]$ component]. Although this mechanism has been extensively investigated, the conversion itself still remains under debate. Type III radio bursts can be observed from metric to kilometric wavelengths (Reiner et al., 2000). From metric to decametric wavelengths we can usually distinguish the $F$ and $H$-components while the $F$-component is often more intense than the $H$-component. At kilometric wavelengths it is problematic to determine the observed component. Kellogg (1980) has developed a method for determination of the component for cases when Type III triggering electron beams intersect the spacecraft.

Propagation of Type III radio bursts from the source to the spacecraft is affected both by refraction in density gradients and scattering by inhomogeneities in the solar wind ranging on all scales from $\approx 100 \mathrm{~km}$ to $\approx 0.3 \mathrm{AU}$. These effects result in the shifted position of a source location, enlargement of the source size, and decrease of the flux density and degree of polarization (Dulk, 2000). Better 
understanding of proper images of radio sources may shed light on physical processes along the path of the exciter electrons in the IP medium.

While previous solar spacecraft used for Type III radio-burst investigation were all spin-stabilized (International Sun/Earth Explorer 3, Ulysses, Wind, etc.), Solar TErrestrial RElations Observatory (STEREO; Kaiser et al., 2008) is the first three-axis stabilized solar mission to observe these waves. With the two identical STEREO spacecraft and their suite of state-of-the-art instruments, we can study mechanisms and sites of solar energetic-particle acceleration in the low corona and the IP medium. The STEREO/Waves instrument enables us to measure radio flux density from decametric to kilometric wavelengths. Therefore we can investigate global distributions of radio sources in the solar wind when an appropriate electron-density model is used.

In this article, we present statistical results on the frequency spectra of Type III radio bursts at long (i.e. from dekametric to kilometric) wavelengths observed by STEREO/Waves. This is the first of two linked articles that summarize our findings on statistical properties of Type III radio bursts. In this article we focus on a flux density and its relation to electron beams producing these bursts. Goniopolarimetric (GP; also referred to as direction-finding) results will be discussed in the second article (Krupar et al., 2014).

In Section 2 we describe the instrumentation and data processing. In Section 3 we analyze two Type III radio bursts and describe our statistical data set. Next we present a statistical examination of Type III radio bursts with focus on the frequency spectra and the model of the flux density as a function of the radial distance. In Section 4 we summarize our findings and we make concluding remarks.

\section{Instrumentation and Methodology}

\subsection{The STEREO/Waves Instrument}

STEREO provides us with first stereoscopic measurements of the solar phenomena using identical instruments onboard (Biesecker, Webb, and St. Cyr, 2008). The two STEREO spacecraft are three-axis stabilized and carry the STEREO/Waves instruments, which measure electric-field fluctuations between $2.5 \mathrm{kHz}$ and $32.025 \mathrm{MHz}$ (Bougeret et al., 2008; Bale et al., 2008). The three monopole antenna elements (six meters long), made from berylliumcopper, are used by STEREO/Waves to measure electric-field fluctuations (Bale et al., 2008). Although we use three orthogonal antennas, their effective antenna directions $\left[\zeta_{\text {eff }}\right.$ and $\left.\xi_{\text {eff }}\right]$ and lengths $\left[l_{\text {eff }}\right]$ are different from the physical ones due to their electrical coupling with the spacecraft body.

For our survey we have used data from the High Frequency Receiver (HFR: a part of the STEREO/Waves instrument; Bougeret et al. 2008), which is a dual-channel receiver (connected to two antennas at one time) operating in the frequency range $125 \mathrm{kHz}-16.025 \mathrm{MHz}$ with a $25 \mathrm{kHz}$ effective bandwidth. HFR has instantaneous GP capabilities between $125 \mathrm{kHz}$ and $1975 \mathrm{kHz}$ allowing us to retrieve the direction of arrival of an incoming electromagnetic wave, its flux, and 
its polarization properties (Cecconi et al., 2008). HFR consists of two receivers: HFR1 $(125 \mathrm{kHz}-1975 \mathrm{kHz}, 38$ frequency channels) and HFR2 $(2025 \mathrm{kHz}-$ 16.025 MHz, 281 frequency channels). HFR1 provides us with auto- and crosscorrelations on all antennas (three monopoles), while HFR2 has retrieved only two auto-correlations (one monopole and one dipole) for most of the time since May 2007.

Using a semiempirical model of electron density $\left[n_{\mathrm{e}}\right]$ in the solar corona and IP medium (Sittler and Guhathakurta, 1999) we can assign particular frequencies to radial distances from the Sun. Consequently STEREO/Waves / HFR allows us to investigate GP properties of radio sources located between $4 R_{\odot}$ and $40 R_{\odot}$ while the intensity is measured up to $1 \mathrm{R}_{\odot}$ above the Sun's surface (Figure 1).

For converting the voltage power spectral density at the terminals of the antennas $\left[V^{2}: \mathrm{V}^{2} \mathrm{~Hz}^{-1}\right]$ into physical units we have applied the method described by Zaslavsky et al. (2011). In order to compute the flux density $\left[S: \mathrm{Wm}^{-2} \mathrm{~Hz}^{-1}\right]$ as below, we need to know the effective antenna lengths and receiver properties.

$$
\begin{gathered}
S=\frac{V^{2}}{Z_{0} \Gamma^{2} l_{\mathrm{eff}}^{2}}, \\
\Gamma=\left|\frac{C_{\mathrm{a}}}{C_{\mathrm{a}}+C_{\mathrm{s}}}\right|,
\end{gathered}
$$

where $Z_{0}=\sqrt{\mu_{0} / \epsilon_{0}} \approx 120 \pi$ denotes the impedance of vacuum. The $\Gamma$-coefficient is the gain factor of the receiver-antenna system and is composed from the antenna capacitance $\left[C_{\mathrm{a}}\right]$ and the stray capacitance $\left[C_{\mathrm{s}}\right]$.

The model of the galactic background brightness $\left[B_{\text {model }}\right]$, as a nearly stable isotropic source, allows us to determine reduced effective antenna lengths $\left[\Gamma l_{\mathrm{eff}}\right]$ according to Zaslavsky et al. (2011):

$$
\begin{gathered}
\Gamma l_{\mathrm{eff}}=\left(\frac{3}{4 \pi Z_{0}} \frac{V^{2}-V_{\text {noise }}^{2}}{B_{\text {model }}}\right)^{1 / 2} \\
B_{\text {model }}=B_{0} f_{\mathrm{MHz}}^{-0.76} e^{-\tau} \\
\tau=3.28 f_{\mathrm{MHz}}^{-0.64}
\end{gathered}
$$

where $B_{0}=1.38 \times 10^{-19} \mathrm{Wm}^{-2} \mathrm{~Hz}^{-1} \mathrm{sr}^{-1}$ and $f_{\mathrm{MHz}}$ is the frequency in $\mathrm{MHz}$ (Novaco and Brown, 1978). The $V_{\text {noise }}^{2}$ represents a square noise generated by the receiver itself and hence should be subtracted before the antenna calibration. We have adapted $V_{\text {noise }}^{2}$ values from Zaslavsky et al. (2011):

- $V_{\text {noise }}^{2}=-160.9 \mathrm{~dB}$ for STEREO-A channel1

- $V_{\text {noise }}^{2}=-162.8 \mathrm{~dB}$ for STEREO-A channel2 $^{2}$

- $V_{\text {noise }}^{2}=-162.4 \mathrm{~dB}$ for STEREO-B channel1

- $V_{\text {noise }}^{2}=-165.9 \mathrm{~dB}$ for STEREO-B channel2 
These values have been obtained by $\chi^{2}$-minimization considering the $V_{\text {noise }}^{2}$ to be frequency independent (Zaslavsky et al., 2011). We have performed the same analysis as Zaslavsky et al. (2011) in order to obtain accurate reduced effective antenna lengths $\left[\Gamma l_{\text {eff }}\right]$ as a function of frequency (Figure 21). These parameters have been retrieved by comparing the lowest $1 \%$ of the data observed within one day (13 January 2007) and the modeled galactic background $\left[V_{\text {galaxy }}^{2}\right]$ using the Equation (3). The low frequency part $[f<750 \mathrm{kHz}]$ is mostly affected by the shot noise induced by solar-wind particles impacting on the antennas (Bougeret et al., 2008) and by the quasi-thermal noise (QTN) produced by the ambient plasma (Meyer-Vernet and Perche, 1989). Since these effects are stronger than the galactic background, the method used is not reliable for retrieving $\Gamma l_{\text {eff }}$ for the low frequency part.

On the other hand, the short dipole approximation for STEREO antennas is not valid for frequencies above $\approx 4 \mathrm{MHz}$ since corresponding wavelengths become comparable with the reduced effective antenna length $\left[\Gamma l_{\text {eff }}\right]$ and thus induced voltages are not proportional to electric field fluctuations (Bale et al., 2008). In Figure 2 we can identify several frequency channels (24 for STEREO-A and 26 for STEREO-B from a total of 319 , i.e. $7 \%$ ) with larger $\Gamma l_{\mathrm{eff}}$ than their neighboring frequency channels. When the measured signal is significantly larger than the fit of the galactic background model, it is likely that this discrepancy is due to instrumental effects, e.g. frequency interferences (Zaslavsky et al., 2011). We have excluded these outlying values of $\Gamma l_{\text {eff }}$ from our analysis.

For HFR1 $(f<2 \mathrm{MHz})$ we have used fixed reduced antenna effective lengths (e.g. see dashed lines in Figure 2 for the $\mathrm{Z}$ antennas). Measured fluxes by the three monopoles have been transformed into the orthogonal frame using effective antenna directions obtained by observations of the non-thermal Auroral Kilometric Radiation (AKR) during STEREO-B roll maneuvers (Krupar et al., 2012). As no AKR has been observed by STEREO-A, the effective directions have been assumed to be the same. The three calculated orthogonal components of the flux density are summed to obtain the total flux density induced by an incident radio wave.

The antenna calibration for HFR2 $(f>2 \mathrm{MHz})$ is more complex, as two effects play a part, resulting in the reduced antenna effective length being larger than the physical one for higher frequencies above $\approx 12 \mathrm{MHz}$ (Zaslavsky et al., 2011). The first effect is the half-wave resonance that changes the effective antenna formulas to be functions of frequency. The second one is the increase of $\Gamma$ above one due to the electrical-circuit resonance as the antenna becomes inductive $\left(C_{\mathrm{a}} \rightarrow-C_{\mathrm{s}}\right)$. Above $12 \mathrm{MHz}$ the reduced effective antenna length rapidly changes and the conversion to physical units according to Equation (11) becomes inaccurate. For HFR2 we have used reduced antenna effective lengths from Figure 2 as a function of frequency. We have summed both contributions of the $\mathrm{X}-\mathrm{Y}$ dipole and the Z-monopole to obtain the total flux density.

In order to improve the signal-to-noise ratio we have subtracted receiver background levels from the data before we performed our analysis. These levels have been calculated as the median values over a period of 15 days ( 7 before, the corresponding one, and 7 after) of the given auto-correlation for each channel-antenna configuration separately. 


\section{Results and Discussion}

We have manually selected 152 time-frequency intervals when Type III radio bursts have been observed by STEREO/Waves between May 2007 and February 2013. The separation angle between spacecraft in the ecliptic plane ranged between $7^{\circ}$ (May 2007) and $180^{\circ}$ (February 2011). We have included only simple and isolated events when flux density was intense enough for the GP analysis.

\subsection{May 2009 Type III Radio Burst}

As an example from our list of events we present a Type III radio burst observed on 6 May 2009 by both STEREO spacecraft (Figure 3). A faint A3.8 X-ray flare located at $\mathrm{N} 20^{\circ} \mathrm{E} 65^{\circ}$ triggered the Type III radio burst. During this event STEREO-A was at $48.2^{\circ}$ West from the Sun-Earth line at 0.96 AU from the Sun whereas STEREO-B was located at $46.9^{\circ}$ East and 1.02 AU from the Sun. The separation angle between the STEREO spacecraft in the ecliptic plane was $95^{\circ}$. STEREO-B, located near the source by $18.1^{\circ}$ longitude, observed the Type III radio burst to have been much intense than STEREO-A being at $113.2^{\circ}$ longitude from the source. Also the spectral shape of the emission at STEREO$\mathrm{B}$ is much broader than at STEREO-A. The onset time of the Type III radio burst was observed about one minute earlier at STEREO-B than at STEREO-A. Whilst the frequency cutoff was not observed at STEREO-B, the radio burst is detected between $325 \mathrm{kHz}$ and $5 \mathrm{MHz}$ only at STEREO-A.

As we do not observe the triggering electron beam, we are unable to use the method of Kellogg (1980) to distinguish the $F$ - and $H$-components of the Type III radio burst. When both components are observed, the $F$-component has typically larger flux density than the $H$-component (Dulk et al., 1998). Using Monte Carlo simulations, Thejappa, MacDowall, and Kaiser (2007) showed that scattering due to random density fluctuations at $120 \mathrm{kHz}$ extends the visibility of the $F$-and $H$-components from $\approx 18^{\circ}$ to $\approx 90^{\circ}$ and from $\approx 80^{\circ}$ to $\approx 150^{\circ}$, respectively. In other words, the $F$-component is visible only in a narrow cone from a source only when compared to $H$-component being visible almost everywhere. From spectral shapes and relative positions between the spacecraft and the flare site, we may conclude that STEREO-B most probably observed the $F$ and $H$-components whereas STEREO-A measured the $H$-component only.

Figure 4 displays peak fluxes as a function of frequency for STEREO-A (on the left) and STEREO-B (on the right). A signal detected at STEREO-B is about $100 \times$ larger than at STEREO-A. It is in agreement with position of the solar flare site, detected a lower signal at STEREO-A (Figure 4), and an observed difference of onset times between the two spacecraft (Figure 3). The maximum flux density at both spacecraft occurs at $\approx 1.5 \mathrm{MHz}$.

We may identify a discontinuity in the HFR1/HFR2 connection at $2 \mathrm{MHz}$ on STEREO-A but not on STEREO-B.

\subsection{November 2011 Type III Radio Burst}

As another example we present a Type III radio burst observed on 23 November 2011 by the two STEREO spacecraft. STEREO-A was at $106.1^{\circ}$ West from the 
Sun-Earth line at $0.97 \mathrm{AU}$ from the Sun while STEREO-B was located $105.4^{\circ}$ East at 1.09 AU from the Sun. The separation angle between the STEREO spacecraft in the ecliptic plane was $149^{\circ}$. Figure 5 shows flux density $[S]$ from STEREO-A and STEREO-B when an intense Type III radio burst was detected at around 15:55 UT. We have not linked this event to any solar flare as the active region was probably located on the far side of the Sun from the Earth's perspective. The radio burst covers the full frequency range at STEREO-A. STEREO-B observed the emission above $275 \mathrm{kHz}$.

Figure 6] displays peak fluxes as a function of frequency for STEREO-A (on the left) and STEREO-B (on the right). A signal detected at STEREO-A is about ten times larger than at STEREO-B. The maximum flux density occured at $625 \mathrm{kHz}$. We observed increase of the flux above $8 \mathrm{MHz}$ at both spacecraft. This increase can be caused by the antenna calibration when we are close to the half wave resonance. Moreover, some additional calibration effects may take place here. We have decided to keep such events in our data set since this flux increase might be a real feature of Type III radio bursts.

We do not identify any discontinuity in the HFR1/HFR2 connection at $2 \mathrm{MHz}$, which suggests that we can combine measurements of the monopole mode (HFR1) with the dipole/monopole mode (HFR2).

\subsection{Data Set}

Table 1 summarizes Type III radio bursts included in our statistical survey. Numbers of events from both spacecraft are about the same. For the reasons described in Section 3.1 we do not distinguish $F / H$ components at long wavelengths in our data set. Generally, the $F$ component is more intense and more directive when compared to the $H$ component (Dulk, 2000; Thejappa, MacDowall, and Kaiser, 2007). These two opposing effects cause uncertainty which of the two components is predominant in our data set. Therefore, we will discuss our results considering that both plasma emission processes take place.

The topmost panel of Figure 7 displays the histogram of the observed Type III radio bursts vs time at STEREO-A and STEREO-B. The middle panel is the separation angle between STEREO-A and STEREO-B. The last panel contains absolute measurements of flux density from the Sun at a wavelength of 10.7 centimeters averaged over the month measured on the ground (www.spaceweather.gc.ca). This value can be used as an estimator of the solar activity. Although the Sun exhibited increased activity as of 2011 we do not have many events from this period since we include only simple and isolated emissions.

We have compared onset times of Type III radio bursts with onset times of solar flares listed by the Lockheed Martin Solar and Astrophysics Laboratory (www.Imsal.com/solarsoft/latest_events_archive.html). We have been able to link only 31 bursts with solar flares (from total of 152 , i.e. $20 \%$ of all events). Since $\mathrm{X}$-ray imagers are located close to the Earth, we have more linked events in 2007 (up to $45 \%$, when the two STEREO were near the Earth) compared to 2012-2013 when only few Type III radio bursts have been associated (the two STEREO spacecraft were on the far side of the Sun). 
Figure 8 shows a histogram of solar flares responsible for the aforementioned 31 Type III radio bursts (flare class A: 6 events, flare class B: 15 events, flare class C: 9 events, flare class M: 1 event). Generally, more intense solar flares trigger complex Type III radio bursts which are not the area of our interest.

We have also compared events from our data set with ground-based observations. The results will be addressed in a separate publication.

\subsection{Frequency Distribution}

Figure 9 displays histograms of frequencies of all Type III radio bursts from our data set for STEREO-A (solid line) and STEREO-B (dotted line). When combined with the number of events (Table 1) it demonstrates the number of data points used in our survey. Although $f_{\text {pe }}$ at STEREO is typically around $30 \mathrm{kHz}$, only $35 \%$ (STEREO-A) and $25 \%$ (STEREO-B) of events have been measured up to $125 \mathrm{kHz}$ corresponding to the lowest-frequency channel of HFR. Our statistical results on low-frequency cutoffs are generally comparable with previous studies dedicated to Wind and Ulysses observations, e.g. by Leblanc et al. (1996) and Dulk et al. (1996). Leblanc, Dulk, and Hoang (1995) suggest that the lowfrequency cutoff can be explained as i) an intrinsic characteristic of the radiation mechanism, ii) an effect of a directivity of the radiation, and iii) propagation effects between the source and the observer.

On the other hand more than $40 \%$ of Type III radio bursts are observed up to $16 \mathrm{MHz}$, which corresponds to a source position located $\approx 1 \mathrm{R}_{\odot}$ and $\approx 1.6 \mathrm{R}_{\odot}$ above the Sun's surface assuming the $F$ - and $H$-emission, respectively (Figure 1). Concerning high-frequency cutoffs, Dulk (2000) investigated 269 events observed by the Wind spacecraft and $70 \%$ of them started at frequencies below $13.8 \mathrm{MHz}$ - the maximum frequency recorded by the instrument. In our dataset we have around $50 \%$ of radio bursts that commence at frequencies below $13 \mathrm{MHz}$.

\subsection{Statistical Analysis of the Frequency Spectra}

We have identified frequencies corresponding to the maximum-flux density for each Type III radio burst separately. Table 2 contains statistical distributions of these peak frequencies. Medians of peak frequencies are around $1 \mathrm{MHz}(825 \mathrm{kHz}$ at STEREO-A and $925 \mathrm{kHz}$ at STEREO-A) which is in agreement with previous observations. Weber (1978) found in data from the Interplanetary Monitoring Platform-H (IMP-6) spacecraft $(30-9900 \mathrm{kHz})$ that the maximum flux density occurs at $\approx 1 \mathrm{MHz}$.

We have investigated median values of the flux density $S$ vs. frequency (see Figure 10). As the distribution has a log-normal character, we have used median values instead of mean ones. The maximum flux density $\left(3 \times 10^{-18} \mathrm{Wm}^{-2} \mathrm{~Hz}^{-1}\right.$ or $3 \times 10^{4} \mathrm{sfu}$ ) occurs at $\approx 1 \mathrm{MHz}$ on both spacecraft. The low-frequency part is affected by the QTN and shot noise, especially for STEREO-A. We have observed a slight increase of flux at STEREO-A for frequencies above $10 \mathrm{MHz}$ but not at STEREO-B. In the case of STEREO-B, it is covered by noise as the 75th percentile exhibits the same trend (Figures 4 and 6). This increase can be caused by inconsistencies of the antenna calibration when we are close to the half-wave 
resonance (Figure 2). On the other hand it can be a real feature of Type III radio bursts. Unfortunately, we cannot compare our results with previous studies since this frequency range $(8 \mathrm{MHz}-16 \mathrm{MHz})$ has not been investigated by spaceborne nor ground-based instruments. Therefore we conclude that measurements of frequencies above $8 \mathrm{MHz}$ should be considered carefully.

\subsection{Interpretation of the $1 \mathrm{MHz}$ maximum}

Using the electron-density model of the solar wind we can determine average distances of radio sources from the Sun of Type III radio bursts (Figure 1). A frequency of $1 \mathrm{MHz}$ corresponds to a radio source located at $\approx 8 \mathrm{R}_{\odot}$ and $\approx 14 \mathrm{R}_{\odot}$ from the Sun for the $F$ - and $H$-component, respectively (dashed and dotted lines in Figure 1). The plasma density $[n(r)]$ in the corona decreases faster than $r^{-2}$, but starting from around $7 \mathrm{R}_{\odot}$ it decreases as $r^{-2}$ (a solid line in Figure 1). The $1 \mathrm{MHz}$ maximum in Figure 10 coincides with this region. One should note that the critical radius of Parker's model of the solar-wind expansion is typically about $10 \mathrm{R}_{\odot}$ being roughly between $8 \mathrm{R}_{\odot}$ and $14 \mathrm{R}_{\odot}$.

We have developed a simplified analytical model of the flux density as a function of radial distance. Let us consider propagation and generation of plasma waves in the corona. Assuming fast relaxation of the beam, we find that the beam propagates as a beam-plasma structure (e.g. Kontar, 2001), so that the $1 \mathrm{D}$ electron distribution function $[f(x, v, t)]$ is

$$
\begin{aligned}
f(x, v, t) & =\frac{n_{\mathrm{b}}}{n_{0}} \exp \left(-\frac{\left(x-v_{0} t / 2\right)^{2}}{d^{2}}\right), v<v_{0} \\
& =0, v>v_{0},
\end{aligned}
$$

and the spectral energy density of Langmuir waves with wavenumber $k=\omega_{p e} / v$ is

$$
W(x, v, t)=\frac{m_{\mathrm{e}} n_{\mathrm{b}}}{v_{0} \omega_{\mathrm{pe}}} v^{4}\left(1-\frac{v}{v_{0}}\right) \exp \left(-\frac{\left(x-v_{0} t / 2\right)^{2}}{d^{2}}\right), v<v_{0},
$$

where $f(x, v, t)$ is the plateau-like electron distribution function of the beam electrons, $n_{\mathrm{b}}$ their number density, $v_{0}$ is the maximum velocity of the plateau electrons, $m_{\mathrm{e}}$ is electron mass, and $W(x, v, t)$ is the 1D Langmuir-Waves spectral density. Let us first consider emission at the spatial peak, e.g. $x \approx v_{0} t / 2$ and near the spectral peak $v \approx 4 / 5 v_{0}$ :

$$
W(x, v, t) \simeq \frac{n_{\mathrm{b}}}{\omega_{p e}} v_{0}^{3} .
$$

In a case where of the $H$-component of an electromagnetic (EM) radiation is saturated we consider its spectral energy density $\left[W_{H}^{\mathrm{EM}}\right]$ to be proportional to the energy of Langmuir waves (Melrose, 1980):

$$
W_{H}^{\mathrm{EM}}(x, v, t) \simeq W(x, v, t) .
$$

The spectral flux density of the $H$-component at the Earth $\left[S_{H}\right]$ can be estimated (the same as if the spacecraft is at $1 \mathrm{AU}$ ): 


$$
S_{H}=W_{H}^{\mathrm{EM}} \frac{\mathrm{d} k}{\mathrm{~d} \omega} A v_{\mathrm{g}} \frac{1}{4 \pi R_{\phi}^{2}},
$$

where $\mathrm{d} k / \mathrm{d} \omega=1 / v_{\mathrm{g}}, v_{\mathrm{g}}$ is the group velocity of the EM waves, $A$ is the area of the source, and $R_{\phi}$ is the $1 \mathrm{AU}$ distance. Hence one finds, ignoring constants:

$$
S_{H} \propto \frac{n_{\mathrm{b}}}{f_{\mathrm{pe}}} v_{0}^{3} A,
$$

where $f_{\mathrm{pe}}$ is the plasma frequency. Further assuming that $n_{\mathrm{b}} A v_{0}^{3} \propto r^{-2}$, one obtains

$$
S_{H} \propto \frac{1}{r^{2}} \frac{1}{f_{\mathrm{pe}}} .
$$

We have included the model in arbitrary units in Figure 10, The semiempirical model of electron density of Sittler and Guhathakurta (1999) in the solar corona and IP medium has been used for estimating $f_{\text {pe }}$. The model of the flux density as a function of frequency $\left[S_{H}\left(f=2 f_{\mathrm{pe}}\right)\right]$ exhibits a maximum at $4 \mathrm{MHz}$ (corresponding to a radio source located at $5 \mathrm{R}_{\odot}$ from the Sun's center) that differs from the observation (maximum at $1 \mathrm{MHz}$ ). The model assumes the $H$-emission only since a relation between the spectral energy density for the $F$-emission $W_{F}^{\mathrm{EM}}$ and the energy of Langmuir waves $[W]$ is not obvious.

However, we have modified the model for the $F$-component considering that the radio flux is proportional between the two components $\left[S_{F}\left(f=f_{\mathrm{pe}}\right)\right]$. In this case we have obtained a maximum at $2 \mathrm{MHz}$ being in a better agreement with the observed maximum at $1 \mathrm{MHz}$. One should note that this approach is very simplified omitting many physical processes such as an efficiency of Langmuir and radio waves conversion, volume of source regions, and scattering of radio beams by density fluctuations.

\section{Summary and Concluding Remarks}

Type III radio bursts belong among the most intense electromagnetic emissions in the heliosphere. They can be observed from tens of $\mathrm{kHz}$ up to several $\mathrm{GHz}$. They are frequently detected by STEREO/Waves instruments, which provide us with a unique stereoscopic view of radio sources in a frequency range from $125 \mathrm{kHz}$ to $16 \mathrm{MHz}$. These frequencies correspond to radial distances from the Sun from $1 \mathrm{R}_{\odot}$ to $50 \mathrm{R}_{\odot}$ and $1.6 \mathrm{R}_{\odot}$ to $85 \mathrm{R}_{\odot}$ for the $F$ - and $H$-component, respectively.

We have investigated properties of 152 simple and isolated Type III radio bursts observed by the two STEREO spacecraft between May 2007 and February 2013. We show a detailed analysis of two events from our data set, as an example. Our main statistical results on the frequency spectra are as follows:

i) We have observed the low-frequency cutoff at $125 \mathrm{kHz}$ in $65 \%-75 \%$ of events. 
ii) More than $40 \%$ of Type III radio bursts are observed up to $16 \mathrm{MHz}$, which corresponds to a source position located $\approx 1 \mathrm{R}_{\odot}$ and $\approx 1.6 \mathrm{R}_{\odot}$ from the Sun's surface for the $F$ - and $H$-component, respectively.

iii) We have found that the maximum of flux density $\left(S \approx 3 \times 10^{-18} \mathrm{Wm}^{-2} \mathrm{~Hz}^{-1}\right)$ occurs at $1 \mathrm{MHz}$ on both spacecraft which corresponds to radio sources located at $8 \mathrm{R}_{\odot}$ and $14 \mathrm{R}_{\odot}$ for the $F$ - and $H$-component, respectively.

iv) We have introduced a simple model of the radio emission flux density vs. radial distance which provides us with a peak at a frequency within a factor of four (the $H$-component) and two (the $F$-component) from the observation, respectively.

The observed low- high-frequency cutoffs can be explained by intrinsic features of radio sources, their directivity, and/or propagation effects between the source and the spacecraft. The source region of radio emissions at $1 \mathrm{MHz}$ corresponds to the region where a change of the plasma density gradient takes place.

We have also observed an increase of the flux density above $8 \mathrm{MHz}$, which corresponds to radial distances from the Sun of $1.6 \mathrm{R}_{\odot}$ and $2.6 \mathrm{R}_{\odot}$ for the $F$ and $H$-component, respectively. We conclude that results of the radio flux above $8 \mathrm{MHz}$ are questionable probably due to instrumental effects.

The model for the $H$-component exhibits a peak at $4 \mathrm{MHz}$. We have modified the model for the $F$-component and the obtained maximum at $2 \mathrm{MHz}$ better corresponds to the maximum observed in the data. One should note that various assumptions on an electron density profile in the solar wind, variations of an exciter speed, and an electron beam density profile may lead to different maximum locations.

In the future we plan to perform simulations of the resonant interaction of an electron beam with Langmuir waves in connection with the observed $1 \mathrm{MHz}$ maximum.

\section{Acknowledgements}

The authors would like to thank the many individuals and institutions who contributed to making STEREO/Waves possible. J. Soucek acknowledges the support of the Czech Grant Agency grant GAP209/12/2394. O. Kruparova thanks the support of the Czech Grant Agency grant GP13-37174P. Financial support by STFC and by the European Commission through the "Radiosun" (PEOPLE-2011-IRSES-295272) is gratefully acknowledged (E.P. Kontar). O. Santolik and V. Krupar acknowledge the support of the Czech Grant Agency grant GAP205/10/2279.

\section{References}

Bale, S.D., Ullrich, R., Goetz, K., Alster, N., Cecconi, B., Dekkali, M., Lingner, N.R., Macher, W., Manning, R.E., McCauley, J., Monson, S.J., Oswald, T.H., Pulupa, M.: 2008, The Electric Antennas for the STEREO/WAVES Experiment. Space Sci. Rev. 136, 529. DOI] ADS 
Table 1. The number of radio bursts detected at STEREO-A and at STEREO-B are in the first and second row, respectively. The third row contains a count of simultaneously observed events. The fourth and fifth rows show events that have been observed only by one spacecraft. The total number of Type III radio bursts in our survey is in the last row.

\begin{tabular}{lr}
\hline STEREO-A events & 135 \\
STEREO-B events & 127 \\
\hline STEREO-A and STEREO-B & 110 \\
STEREO-A and not STEREO-B & 25 \\
STEREO-B and not STEREO-A & 17 \\
\hline Total number of events & $\mathbf{1 5 2}$ \\
\hline
\end{tabular}

Table 2. Statistical properties of peak frequencies of Type III radio bursts included in the survey.

\begin{tabular}{lrr}
\hline & STEREO-A & STEREO-B \\
\hline & & \\
Mean $[\mathrm{kHz}]$ & 1291 & 1498 \\
STD [kHz] & 1534 & 1894 \\
Median $[\mathrm{kHz}]$ & 825 & 925 \\
25th percentile $[\mathrm{kHz}]$ & 525 & 625 \\
75th percentile $[\mathrm{kHz}]$ & 1575 & 1875 \\
\hline
\end{tabular}

Biesecker, D.A., Webb, D.F., St. Cyr, O.C.: 2008, STEREO Space Weather and the Space Weather Beacon. Space Sci. Rev. 136, 45. DOI ADS

Bougeret, J.L., Goetz, K., Kaiser, M.L., Bale, S.D., Kellogg, P.J., Maksimovic, M., Monge, N., Monson, S.J., Astier, P.L., Davy, S., Dekkali, M., Hinze, J.J., Manning, R.E., AguilarRodriguez, E., Bonnin, X., Briand, C., Cairns, I.H., Cattell, C.A., Cecconi, B., Eastwood, J., Ergun, R.E., Fainberg, J., Hoang, S., Huttunen, K.E.J., Krucker, S., Lecacheux, A., MacDowall, R.J., Macher, W., Mangeney, A., Meetre, C.A., Moussas, X., Nguyen, Q.N., Oswald, T.H., Pulupa, M., Reiner, M.J., Robinson, P.A., Rucker, H., Salem, C., Santolik, O., Silvis, J.M., Ullrich, R., Zarka, P., Zouganelis, I.: 2008, S/WAVES: The Radio and Plasma Wave Investigation on the STEREO Mission. Space Sci. Rev. 136, 487. DOI ADS

Cecconi, B., Bonnin, X., Hoang, S., Maksimovic, M., Bale, S.D., Bougeret, J.-L., Goetz, K., Lecacheux, A., Reiner, M.J., Rucker, H.O., Zarka, P.: 2008, STEREO/Waves Goniopolarimetry. Space Sci. Rev. 136, 549. DOI ADS.

Drummond, W., Pines, D.: 1962, Non-Linear Stability of Plasma Oscillations. Nuclear Fusion 3, 1049.

Dulk, G.A.: 2000, Type III Solar Radio Bursts at Long Wavelengths. Geophys. Mono. Ser., $A G U$ 119, 115. ADS.

Dulk, G.A., Goldman, M.V., Steinberg, J.L., Hoang, S.: 1987, The speeds of electrons that excite solar radio bursts of type III. Astron. Astrophys. 173, 366. ADS

Dulk, G.A., Leblanc, Y., Bougeret, J.-L., Hoang, S.: 1996, Type III bursts observed simultaneously by Wind and Ulysses. Geophys. Res. Lett. 23, 1203. DOI ADS. 


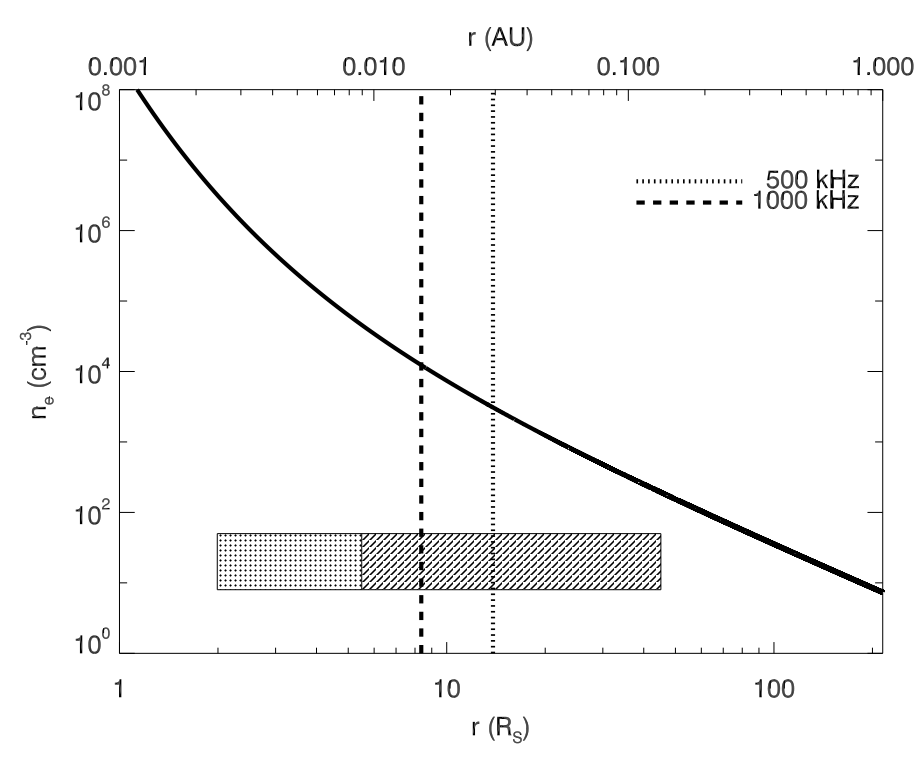

Figure 1. Electron density model vs. distance from the Sun for average solar wind parameters adapted from Sittler and Guhathakurta (1999). The dashed region indicates frequencies where the STEREO/Waves/HFR provides us with the GP data while in the dotted region we can retrieve information on wave intensity only. Dashed and dotted lines indicate radial distances from the Sun where electron density corresponds to a plasma frequency of $1 \mathrm{MHz}$ and $500 \mathrm{kHz}$, respectively.

Dulk, G.A., Leblanc, Y., Robinson, P.A., Bougeret, J.-L., Lin, R.P.: 1998, Electron beams and radio waves of solar type III bursts. J. Geophys. Res. 103, 17223. DOI ADS

Ginzburg, V.L., Zhelezniakov, V.V.: 1958, On the Possible Mechanisms of Sporadic Solar Radio Emission (Radiation in an Isotropic Plasma). Soviet Astron. 2, 653. ADS

Kaiser, M.L., Kucera, T.A., Davila, J.M., St. Cyr, O.C., Guhathakurta, M., Christian, E.: 2008, The STEREO Mission: An Introduction. Space Sci. Rev. 136, 5. DOI ADS

Kellogg, P.J.: 1980, Fundamental emission in three type III solar bursts. Astrophys. J. 236, 696. DOI ADS

Kontar, E.P.: 2001, Dynamics of electron beams in the solar corona plasma with density fluctuations. Astron. Astrophys. 375, 629. DOI ADS

Kontar, E.P., Reid, H.A.S.: 2009, Onsets and Spectra of Impulsive Solar Energetic Electron Events Observed Near the Earth. Astrophys. J. Lett. 695, L140. DOI ADS

Krupar, V., Santolik, O., Cecconi, B., Maksimovic, M., Bonnin, X., Panchenko, M., Zaslavsky, A.: 2012, Goniopolarimetric inversion using SVD: An application to type III radio bursts observed by STEREO. J. Geophys. Res. 117(16), 6101. DOI ADS

Krupar, V., Maksimovic, M., Santolik, O., Cecconi, B., Kruparova, O.: 2014, Statistical Survey of Type III Radio Bursts at Long Wavelengths Observed by the Solar TErrestrial RElations Observatory (STEREO)/Waves Instruments: Goniopolarimetric Properties and Radio Source Locations, Solar Phys., submitted.

Leblanc, Y., Dulk, G.A., Hoang, S.: 1995, The low radio frequency limit of solar type III bursts: Ulysses observations in and out of the ecliptic. Geophys. Res. Lett. 22, 3429. DOI ADS

Leblanc, Y., Dulk, G.A., Hoang, S., Bougeret, J.-L., Robinson, P.A.: 1996, Type III radio bursts observed by ULYSSES pole to pole, and simultaneously by wind. Astron. Astrophys. 316, 406. ADS

Magelssen, G.R., Smith, D.F.: 1977, Nonrelativistic electron stream propagation in the solar atmosphere and type III radio bursts. Solar Phys. 55, 211. DOI ADS

Mel'nik, V.N., Lapshin, V., Kontar, E.: 1999, Propagation of a Monoenergetic Electron Beam in the Solar Corona. Solar Phys. 184, 353. ADS

Melrose, D.B.: 1980, The emission mechanisms for solar radio bursts. Space Sci. Rev. 26, 3. DOI ADS 


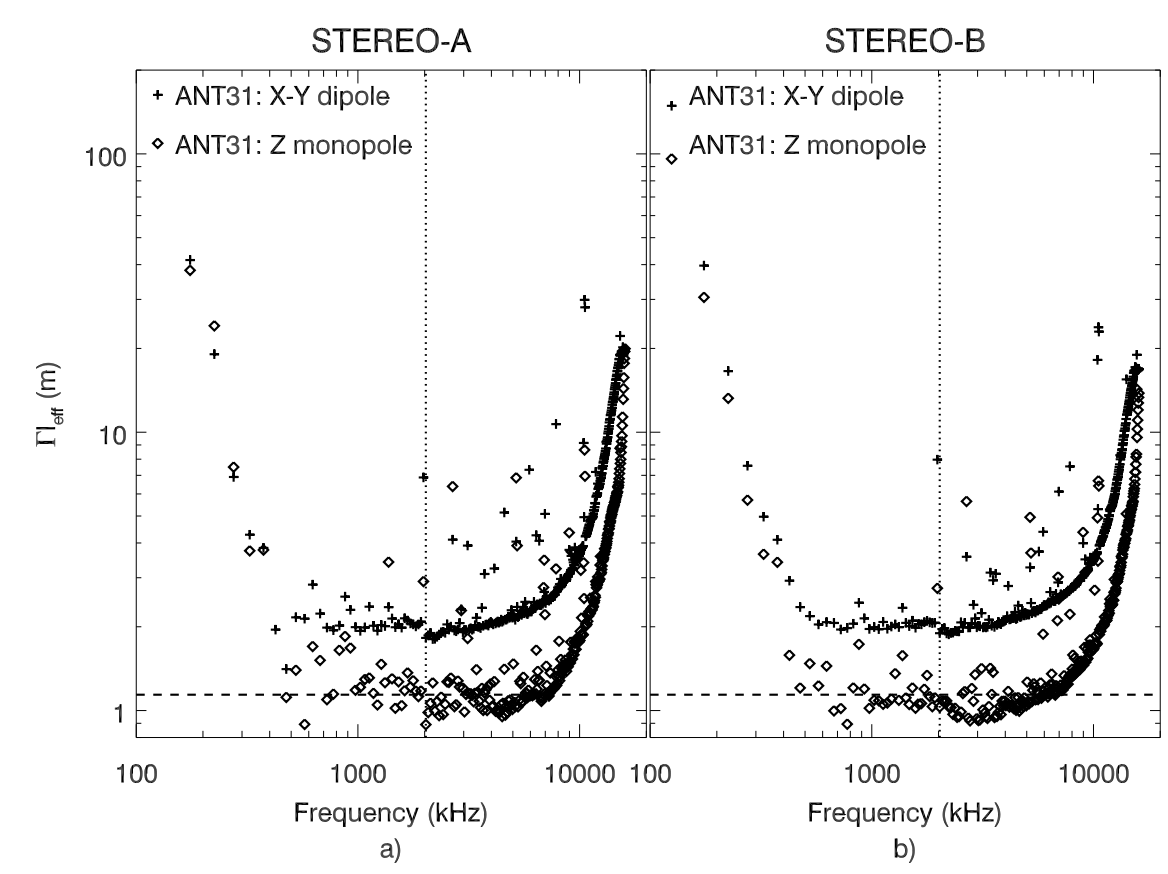

Figure 2. Reduced effective antenna lengths of $\mathrm{X}-\mathrm{Y}$ dipole (crosses) and $\mathrm{Z}$ monopole (diamonds) for STEREO-A (left) and STEREO-B (right) vs. frequency obtained from measurements on January 13, 2007. A black-dotted line separates frequency coverage of HFR1 $(<2 \mathrm{MHz})$ and HFR2 $(>2 \mathrm{MHz})$. A dashed line indicates the $\Gamma l_{\text {eff }}$ of the $\mathrm{Z}$ monopole used for HFR1.

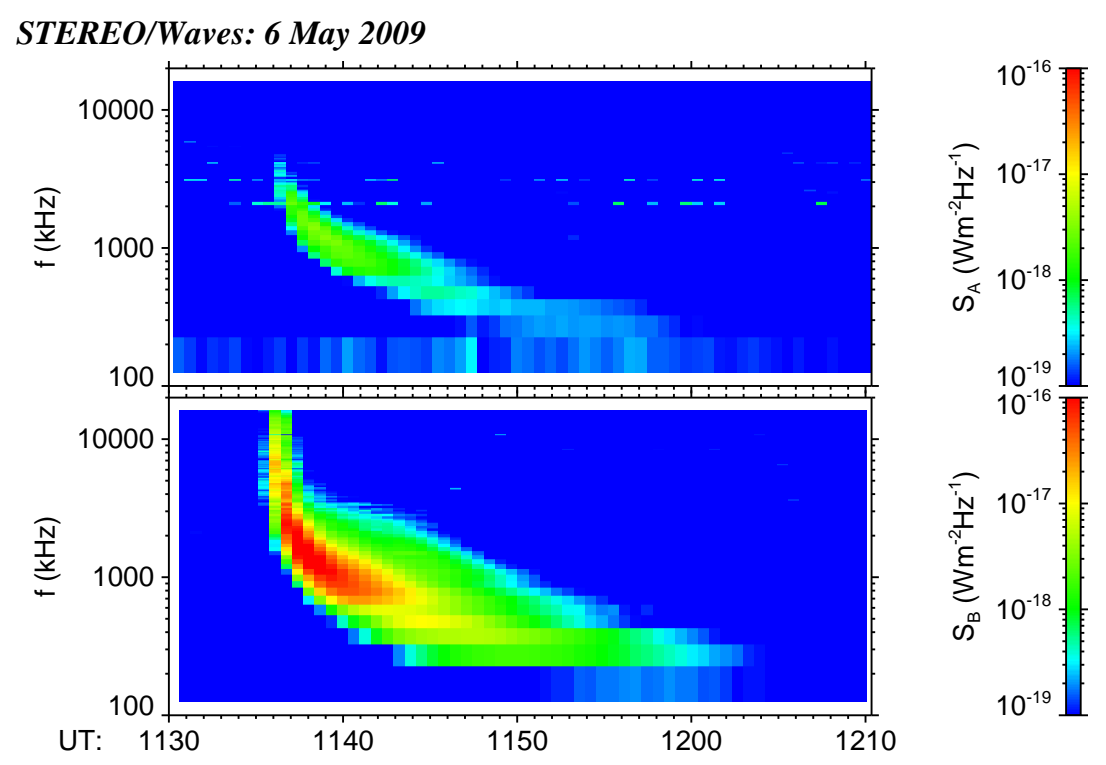

Figure 3. Analysis of measurements recorded from 11:30 to 12:20 UT on 6 May 2009: dynamic spectra for STEREO-A (top) and STEREO-B (bottom). 


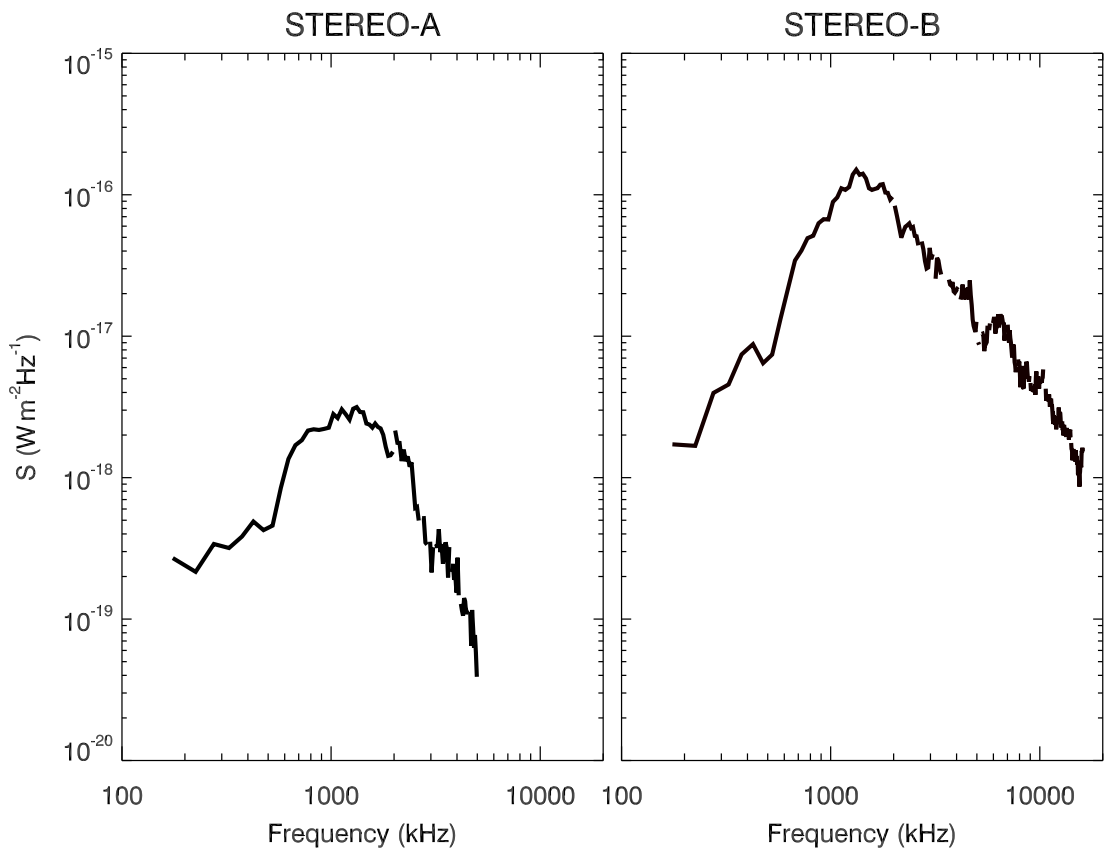

Figure 4. Analysis of measurements recorded from 11:30 to 12:20 UT on 6 May 2009: flux density (peak flux values) vs. frequency for STEREO-A (left) and STEREO-B (right).

STEREO/Waves: 23 November 2011
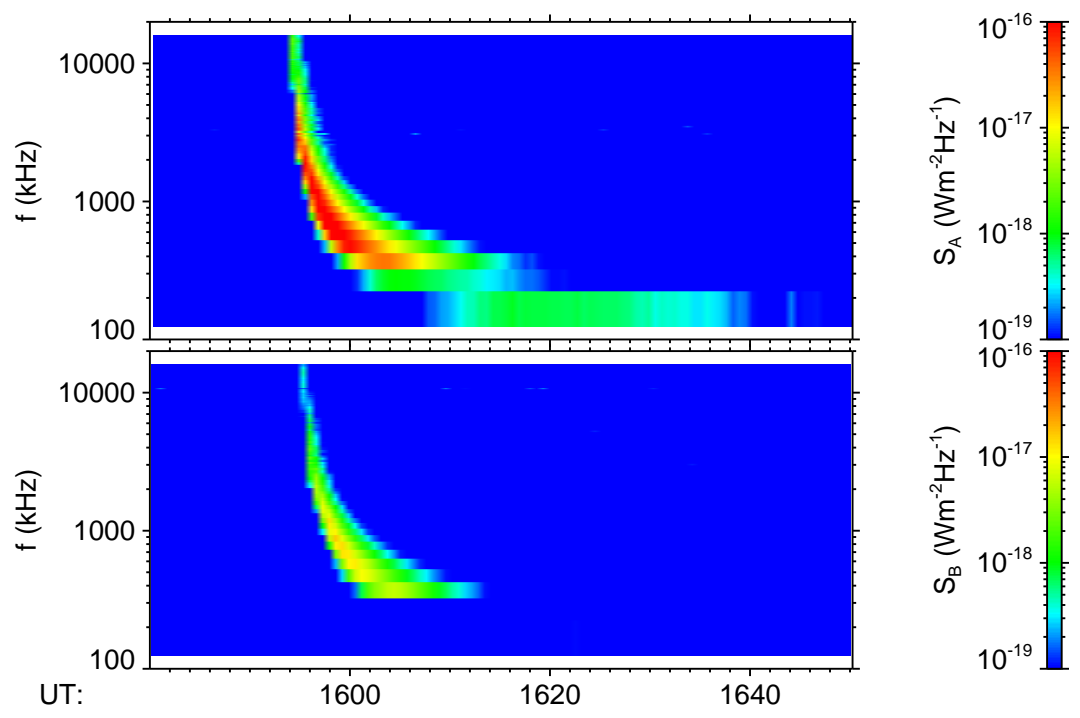

Figure 5. Analysis of measurements recorded from 15:40 to 16:30 UT on 23 November 2011: dynamic spectra for STEREO-A (top) and STEREO-B (bottom). 


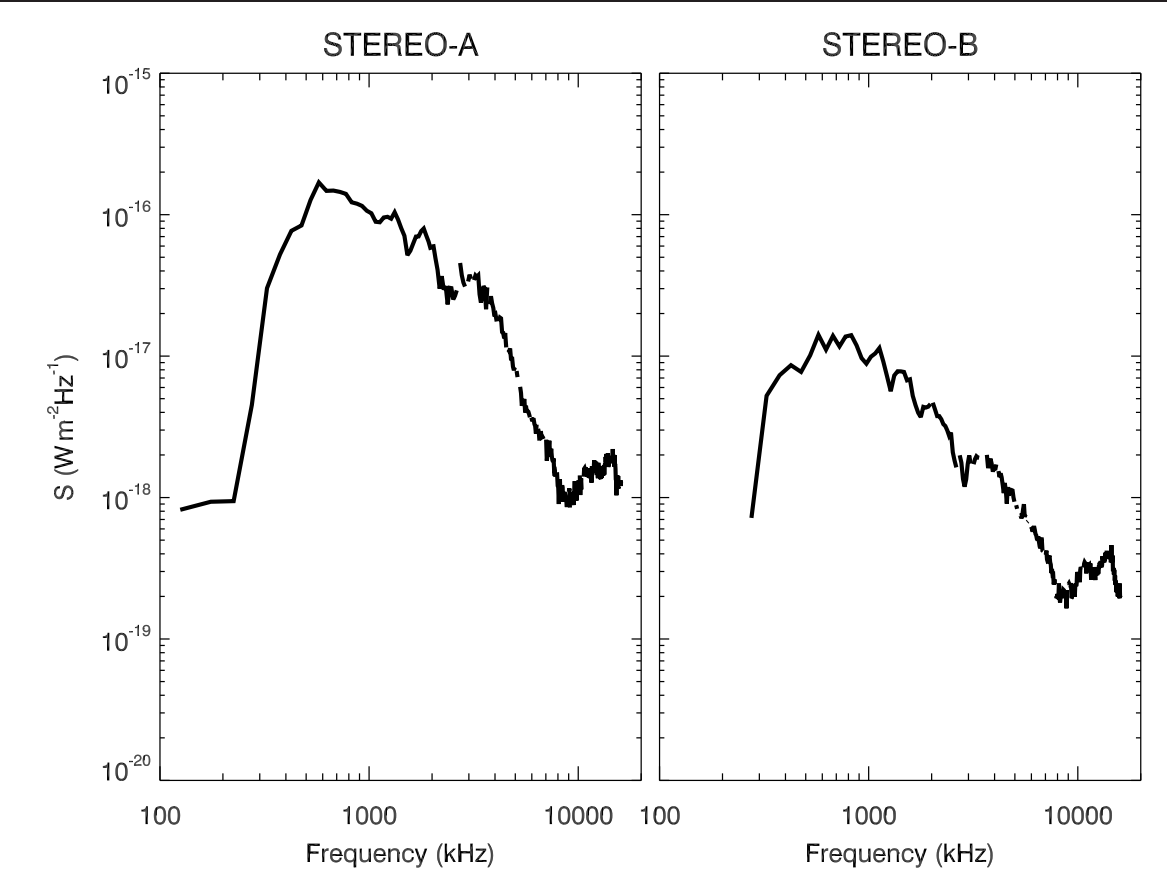

Figure 6. Analysis of measurements recorded from 15:40 to 16:30 UT on 23 November 2011: flux density (peak flux values) vs. frequency for STEREO-A (left) and STEREO-B (on the right).

Melrose, D.B., Cairns, I.H., Dulk, G.A.: 1986, Clumpy Langmuir waves in type III solar radio bursts. Astron. Astrophys. 163, 229. ADS.

Meyer-Vernet, N., Perche, C.: 1989, Tool kit for antennae and thermal noise near the plasma frequency. J. Geophys. Res. 94, 2405. DOI ADS

Novaco, J.C., Brown, L.W.: 1978, Nonthermal galactic emission below 10 megahertz. Astrophys. J. 221, 114. DOI ADS.

Pines, D., Schrieffer, J.R.: 1962, Approach to Equilibrium of Electrons, Plasmons, and Phonons in Quantum and Classical Plasmas. Phys. Rev. E 125, 804. [DOI ADS

Reid, H.A.S., Kontar, E.P.: 2010, Solar Wind Density Turbulence and Solar Flare Electron Transport from the Sun to the Earth. Astrophys. J. 721, 864. DOI ADS

Reiner, M.J., Karlický, M., Jiřička, K., Aurass, H., Mann, G., Kaiser, M.L.: 2000, On the Solar Origin of Complex Type III-like Radio Bursts Observed at and below 1 MHZ. Astrophys. J. 530, 1049. DOI ADS

Robinson, P.A., Cairns, I.H.: 1998, Fundamental and Harmonic Emission in Type III Solar Radio Bursts - III. Heliocentric Variation of Interplanetary Beam and Source Parameters. Solar Phys. 181, 429. DOI ADS

Sittler, E.C. Jr., Guhathakurta, M.: 1999, Semiempirical Two-dimensional MagnetoHydrodynamic Model of the Solar Corona and Interplanetary Medium. Astrophys. J. 523, 812. DOI ADS

Smith, D.F., Sime, D.: 1979, Origin of plasma-wave clumping in type III solar radio burst sources. Astrophys. J. 233, 998. DOI ADS

Thejappa, G., MacDowall, R.J., Kaiser, M.L.: 2007, Monte Carlo Simulation of Directivity of Interplanetary Radio Bursts. Astrophys. J. 671, 894. DOI ADS.

Vedenov, A., Velikhov, E., Sagdeev, R.: 1962, Quasi-Linear Theory of Plasma Oscillations. Nuc. Fus., 465.

Weber, R.R.: 1978, Low frequency spectra of type III solar radio bursts. Solar Phys. 59, 377. DOI ADS

Wild, J.P.: 1950, Observations of the Spectrum of High-Intensity Solar Radiation at Metre Wavelengths. III. Isolated Bursts. Austral. J. Sci. Res. A Phys. Sci. 3, 541. ADS 

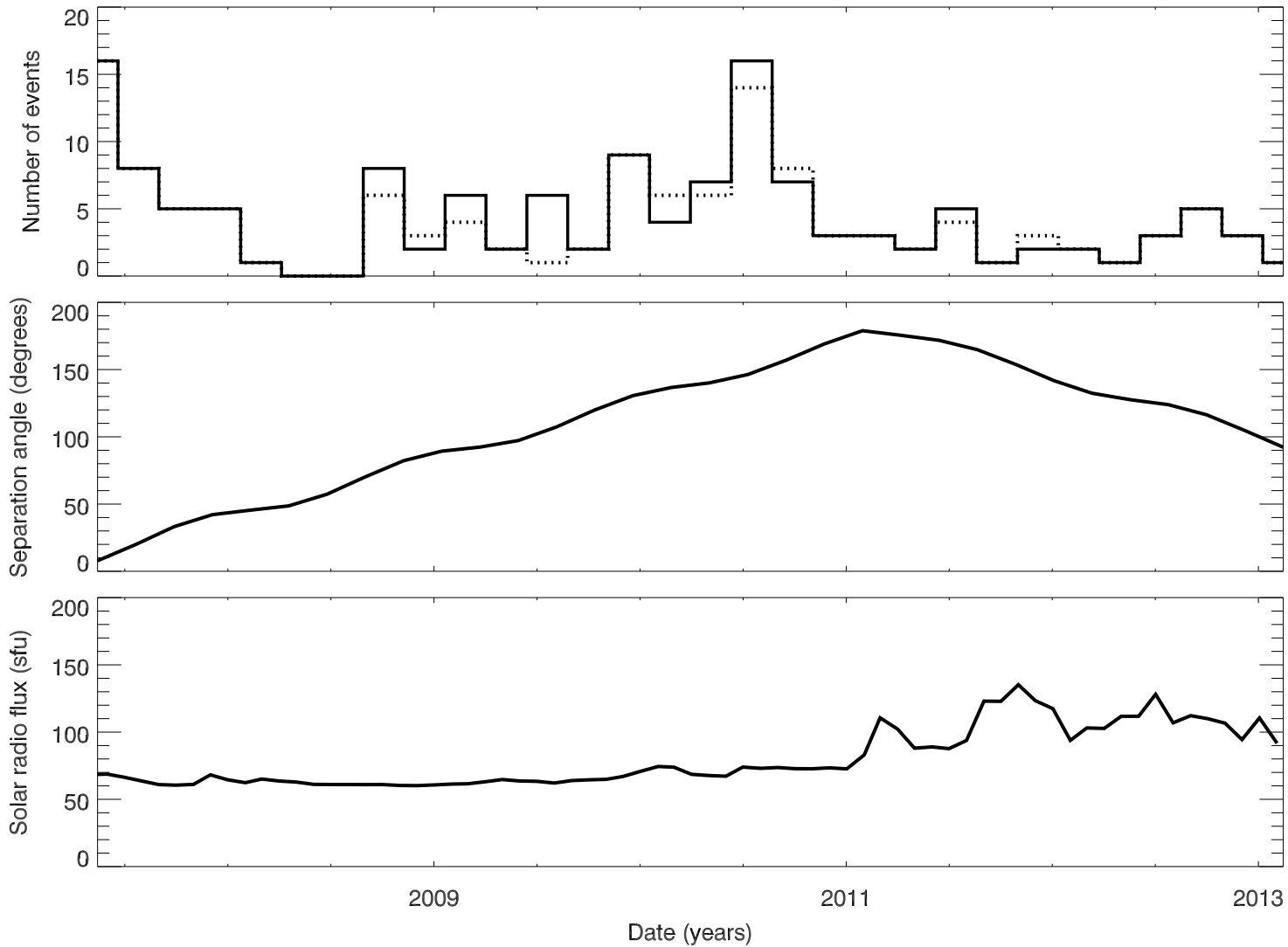

Figure 7. Top panel: histogram of observed Type III radio bursts at STEREO-A (solid line) and STEREO-B (dotted line) between May 2007 and February 2013. Middle: a separation angle between STEREO-A and STEREO-B. Bottom: monthly averages of the absolute value of the solar radio flux $\left(1 \mathrm{sfu} \equiv 10^{-22} \mathrm{Wm}^{-2} \mathrm{~Hz}^{-1}\right)$ retrieved from www.spaceweather.gc.ca

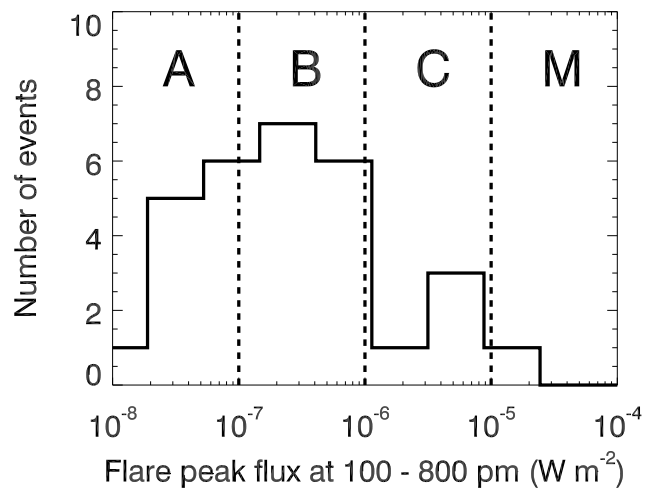

Figure 8. Histogram of solar flares associated with Type III radio bursts. 


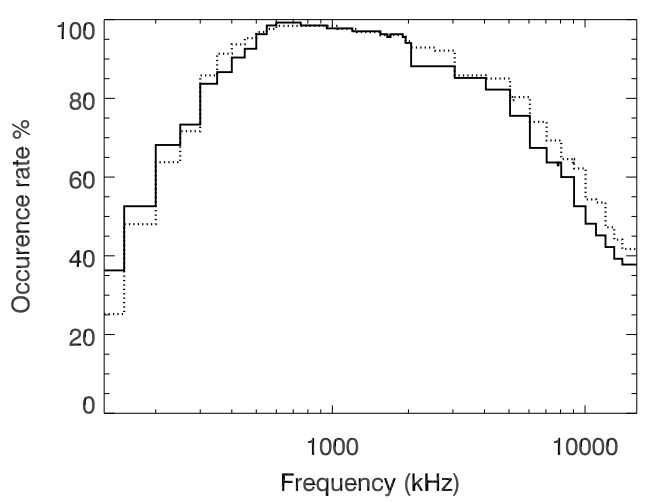

Figure 9. The histogram of frequencies of observed Type III radio bursts at STEREO-A (solid line) and STEREO-B (dotted line).

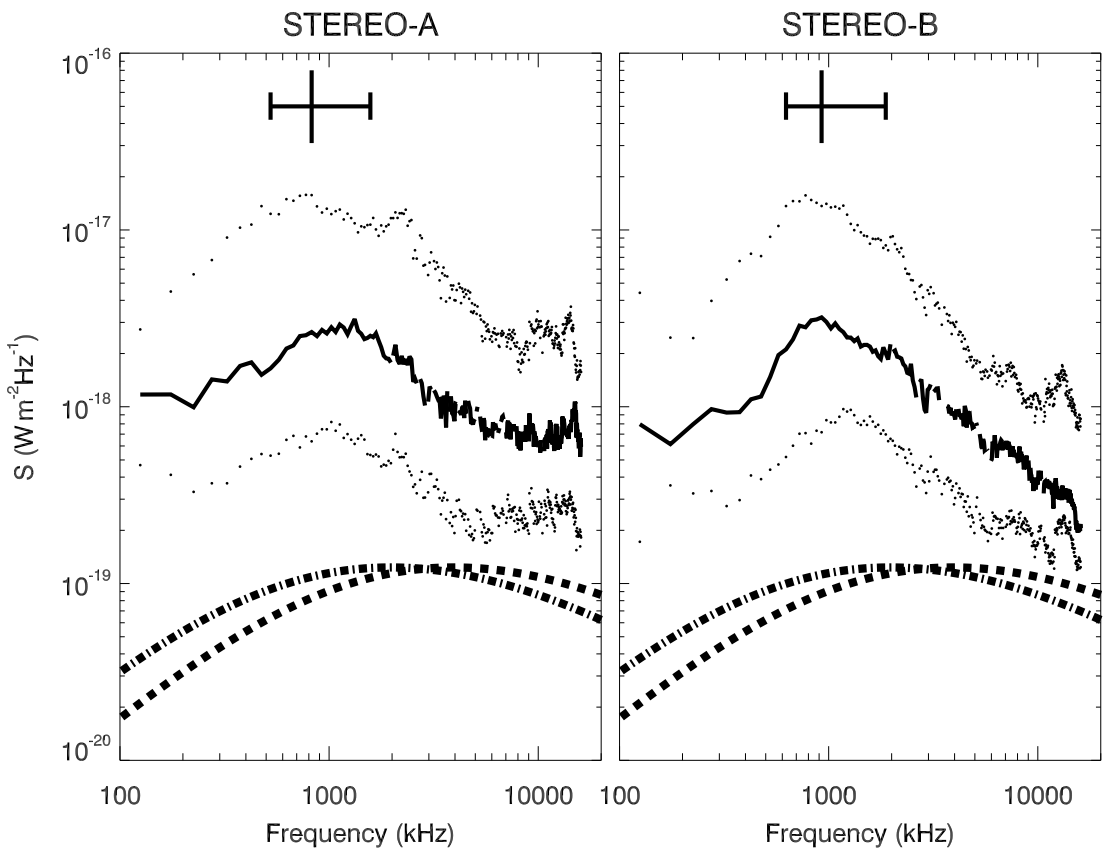

Figure 10. Flux density vs. frequency at STEREO-A and STEREO-B for 152 Type III radio bursts. Solid lines are the medians of the flux for each frequency and dotted lines represent 25 th and 75 th percentiles. Black crosses on the top denote median and 25 th/75th percentiles of the peak frequency from Table 2 Dashed and dashed-dotted lines represent the model of the $H$ - and $F$-component in arbitrary units, respectively.

Zaslavsky, A., Meyer-Vernet, N., Hoang, S., Maksimovic, M., Bale, S.D.: 2011, On the antenna calibration of space radio instruments using the galactic background: General formulas and application to STEREO/WAVES. Radio Sci. 46, RS2008. DOI ADS. 